\title{
Preservation of multidimensional quality of life after endoscopic pituitary adenoma resection
}

\author{
Edward D. McCoul, MD, MPH, ${ }^{4}$ Jeffrey C. Bedrosian, MD, ${ }^{5}$ Olga Akselrod, ${ }^{2}$ Vijay K. Anand, MD, ${ }^{1}$ \\ and Theodore H. Schwartz, MD ${ }^{1,2,3}$
}

\begin{abstract}
1Department of Otolaryngology-Head and Neck Surgery; ${ }^{2}$ Department of Neurological Surgery, Sackler Brain and Spine Center; and ${ }^{3}$ Department of Neuroscience, Feil Brain and Mind Institute, Weill Cornell Medical College, NewYork-Presbyterian Hospital, New York, New York; " Department of Otolaryngology-Head and Neck Surgery, Ochsner Medical Center, New Orleans, Louisiana; and ${ }^{5}$ Department of Surgery, Division of Otolaryngology-Head and Neck Surgery, Maine Medical Center, Portland, Maine
\end{abstract}

OBJECT Pituitary adenomas are well suited to resection by a minimal-access endoscopic technique. Validation of this approach requires prospective outcome studies to determine the impact on quality of life (QOL). This study aims to assess the effect of endoscopic pituitary adenoma resection on site-specific and sinonasal-related QOL before and after endoscopic surgery using validated instruments.

METHODS Consecutive adult patients undergoing endoscopic endonasal resection of pituitary adenoma were prospectively enrolled from a single tertiary care center. All patients completed the Anterior Skull Base Questionnaire (ASBQ) and the 22-Item Sino-Nasal Outcome Test (SNOT-22) preoperatively and then at regular intervals after surgery to assess their perceived QOL with regard to hormonal, surgical, and anatomical factors.

RESULTS Eighty-one of 114 patients were eligible for study; median follow-up was 16 months. This cohort included 24 $(29.6 \%)$ nonsecreting macroadenomas and 57 (70.4\%) hypersecreting tumors. There was significant improvement in the mean ASBQ score at 12 weeks, 6 months, and 1 year after surgery $(p<0.05)$, while postoperative SNOT-22 scores, at the same time points, showed no significant difference from preoperative scores. Both ASBQ and SNOT-22 scores showed transient worsening at 3 weeks postoperatively. Subtotal resection correlated with worse QOL, both overall and among patients with hypersecreting tumors $(p<0.05)$. Extrasellar tumor extension, intraoperative CSF leakage, and a reconstruction technique during surgery did not impact postoperative QOL. Visual disturbances did not significantly alter QOL. There were no postoperative CSF leaks in this series.

CONCLUSIONS Endoscopic resection of pituitary adenoma is associated with long-term improvements in site-specific QOL and stability in sinonasal QOL when assessed pre- and postoperatively with validated instruments. Subtotal resection was the only factor that negatively impacted postoperative QOL. Therefore, gross-total resection should be attempted for all patients to optimize QOL after surgery.

http://thejns.org/doi/abs/10.3171/2014.11.JNS14559

KEY WORDS pituitary adenoma; endoscopic; surgery; quality of life; outcomes research; pituitary surgery

$\mathrm{T}$ HE endoscopic endonasal transsphenoidal approach to the pituitary gland is gaining popularity as an alternative to traditional microscope-assisted techniques. Although still a matter of debate, the endoscopic approach appears to be superior to the microscopic technique with regard to extent of resection, specifically for macroadenomas, $, 12,18$ and also offers benefits for nasal function and morbidity. ${ }^{5,20,21}$ Increased rates of resection can be explained by the improved visualization offered by the endoscope, which is advanced into the nasal cavity to provide a wide panoramic view and a lateral view through the use of straight and angled scopes. Morbidity is theoretically decreased by preserving nasal mucosa, opening the sinuses to permit drainage, avoiding superficial mucosal incisions, and eliminating use of the retractor. ${ }^{5,18,20,21}$ However, controversy remains regarding sinonasal morbidity

ABBREVIATIONS ASBQ = Anterior Skull Base Questionnaire; GH = growth hormone; GTR = gross-total resection; IGF-1 = insulin-like growth factor-1; $Q \mathrm{QOL}=$ quality of life; SNOT-22 = 22-Item Sino-Nasal Outcome Test; STR = subtotal resection.

SUBMITTED March 13, 2014. ACCEPTED November 12, 2014.

INCLUDE WHEN CITING Published online June 5, 2015; DOI: 10.3171/2014.11.JNS14559.

DISCLOSURE The authors report no conflict of interest concerning the material or methods used in this study or the findings specified in this paper. Dr. Schwartz has stock options in Visionsense. 
of the endonasal approach and its impact on quality of life (QOL). In particular, concerns exist over the recent use of nasoseptal flaps to close large skull base defects; these flaps are sometimes used after removal of large pituitary adenomas. Likewise, based on the presumption that surgery is invasive and risky, surgical interventions for benign, slowly evolving pathology should be well tolerated by patients since competing nonsurgical therapies such as radiation or medical therapy may be used. ${ }^{11,13}$

Despite the widespread use of surgery to manage pituitary adenomas, the effect on QOL has been incompletely studied. Quality of life is a patient-reported measure that aims to describe a patient's perception of well-being while eliminating observer bias. Recent interest in outcomes research has highlighted the importance of patient perception in determining the success of a surgical intervention, particularly when the disease is nonmalignant. A surgeon's impression of a patient's well-being following skull base surgery has been shown to be inaccurate, ${ }^{8}$ which demonstrates the need for validated, patient-reported assessments of QOL.

Instruments that measure QOL can either be generalized or be disease- or site-specific and are typically multidimensional. A limited number of studies have sought to examine the effect of endoscopic endonasal pituitary adenoma surgery on sinonasal morbidity, ${ }^{10,30}$ but these studies have not employed instruments specific to the skull base. Other recent studies have relied on generalized QOL measures ${ }^{16,29}$ nonvalidated instruments,${ }^{19}$ or unpaired postoperative data. ${ }^{6,26}$

The primary objective of this study was to determine the effect of endoscopic surgery on patient-reported QOL using validated instruments administered both pre- and postoperatively. Although prior reports have assessed QOL after endoscopic surgery, few studies have directly compared data obtained before and after the intervention. Secondary objectives included determining the effect of endoscopic surgery on sinonasal morbidity, the impact of tumor extension on QOL, and the relationships between visual and endocrine dysfunction and QOL.

\section{Methods}

Consecutive patients who were preparing to undergo endoscopic resection of pituitary adenoma were prospectively enrolled between July 2010 and July 2013 from the outpatient offices of a single tertiary care center. All included patients were adults over 18 years of age. Patients undergoing concurrent craniotomy were excluded, as were emergent cases and those that did not have a complete preoperative assessment. Study approval was obtained from the institutional review board of the Weill Cornell Medical College.

All patients underwent a complete preoperative outpatient evaluation. A complete history taking and physical examination were performed independently by a neurosurgeon and a rhinologic surgeon. Video-assisted nasal endoscopy was performed to identify the presence of an anatomical nasal obstruction or active sinusitis and to rule out intranasal tumor extension. MRI of the brain with gadolinium was performed to assess tumor size using volu- metrics, to identify tumor extension, and to assist in planning the surgical approach. Patients with lesions involving the optic chiasm or optic tract were referred for neuroophthalmological evaluation and visual field testing.

To assess for endocrine derangements, all patients underwent pre- and postoperative endocrinological evaluation of free cortisol, adrenocorticotropic hormone, free thyroxine, thyroid-stimulating hormone, prolactin, growth hormone $(\mathrm{GH})$, insulin-like growth factor-1 (IGF-1), testosterone, estradiol, luteinizing hormone, and folliclestimulating hormone. The diagnosis of a prolactinoma was made when serum prolactin levels were $>150 \mathrm{ng} / \mathrm{ml}$ combined with typical clinical symptoms. In patients with prolactinoma, endocrinological remission was defined as postoperative prolactin levels $<20 \mathrm{ng} / \mathrm{ml}$ in females or $<$ $15 \mathrm{ng} / \mathrm{ml}$ in males. The diagnosis of Cushing disease was based on either abnormal 24-hour urinary free cortisol or abnormal results on low-dose dexamethasone suppression tests, defined as the failure of $1 \mathrm{mg}$ of dexamethasone to reduce plasma cortisol levels to $<1.8 \mathrm{mg} / \mathrm{ml}$ by the next morning. Endocrinological remission of Cushing disease was defined as an early morning cortisol measurement < $1.8 \mathrm{mg} / \mathrm{ml}$ or normalization of the 24-hour urinary free cortisol. The diagnosis of acromegaly was based on abnormal basal fasting levels of GH and IGF-1. Biochemical remission of acromegaly was defined as having a normal IGF-1 level combined with a glucose-suppressed GH level $\leq 0.4 \mathrm{ng} / \mathrm{ml}$ or, alternatively, combined with a basal GH level $\leq 1 \mathrm{ng} / \mathrm{ml}$. The IGF-1 level was always evaluated according to age-adjusted diagrams. Visual dysfunction was determined by neuro-ophthalmological evaluation and included findings of hemianopia or another visual field cut, optic nerve dysfunction, or diplopia due to cranial nerve involvement.

Surgery was performed in accordance with endoscopic operative approaches previously described in the literature. ${ }^{25}$ A multidisciplinary approach between the neurosurgeon and the rhinologic surgeon was used in all cases. All patients underwent repeat MRI of the brain with a navigation protocol to assist with intraoperative localization. Lumbar puncture or lumbar drain placement was performed at the start of each procedure. Lumbar drains were used in patients with tumors $>2.5 \mathrm{~cm}$ in diameter and with $>1 \mathrm{~cm}$ of suprasellar extension. At the start of each procedure, intrathecal fluorescein was injected to localize potential intraoperative leaks of CSF. ${ }^{28}$ Perioperative antibiotics consisted of cefazolin for nonallergic patients. ${ }^{3}$ Rigid straight and angled endoscopes with video-capture capability were used for the entire procedure. Partial inferior turbinectomy and posterior septectomy were performed in all patients to provide improved access for the surgical instrumentation. Following tumor resection, a multilayer reconstruction of the skull base defect was performed in all cases, which may have included a rigid Medpor prosthesis (Stryker), autologous fat, DuraSeal (Covidien), or vascularized flaps for larger tumors. ${ }^{15,22}$ The nasal cavity was filled with Floseal, and a folded Telfa sponge was placed in each nostril to minimize postoperative epistaxis. Postoperative MRI was performed within 24-48 hours after surgery to assess the degree of tumor resection using volumetric measurements and was assessed by a neuroradiologist. 
Two validated QOL measures were used in this study: the Anterior Skull Base Questionnaire (ASBQ) and the 22-Item Sino-Nasal Outcome Test (SNOT-22). Both questionnaires were completed by each patient prior to the preoperative evaluation. Postoperative visits occurred at 3 weeks, 6 weeks, 12 weeks, 6 months, and 1 year following surgery. Each patient was again asked to complete both the ASBQ and the SNOT-22 at each postoperative visit. After completing the surveys, endoscopic examination and intranasal debridement were performed at each visit. At every stage of data collection, survey results were entered into a database by a third party, and the results were unknown to both the neurosurgeon and the rhinologic surgeon.

The ASBQ is a disease-specific QOL instrument that has been validated for use in patients undergoing anterior skull base surgery. ${ }^{79}$ The ASBQ contains 35 items divided into 6 separate QOL domains: performance, physical function, vitality, pain, influence on emotion, and specific symptoms. Responses are recorded on a 5-item Likert scale, with scores ranging from 1 to 5 for each item. Mean subscores are calculated for each domain as well as for the total instrument. Overall score is reported as a mean item score ranging from 1.0 to 5.0, with a higher score indicating a better QOL. While the validation cohort was treated exclusively via open, nonendoscopic surgical approaches, the ASBQ has recently been validated for endoscopic approaches as well. ${ }^{4}$ Multiple studies have demonstrated the validity of the ASBQ for anterior skull base surgery and the association of ASBQ scores with outcomes and other factors including pathology, approach, age, and degree of resection. ${ }^{17}$

The SNOT-22 is a disease-specific instrument designed to assess QOL related to benign sinonasal disease. Although it has not been validated in the setting of pituitary surgery, it was selected for its value in assessing the morbidity that accompanies endoscopic endonasal surgery. It is adapted from prior disease-specific QOL instruments that have been extensively validated in the otolaryngology literature. ${ }^{14,23,24}$ Responses are recorded on a 6-item Likert scale, with scores ranging from 0 to 5 for each item. Values are reported as the mean. Total SNOT-22 scores range from 0 to 110, with a higher score indicating a worse QOL.

The primary outcome measure of this study was the change in ASBQ scores between the preoperative and postoperative visits. Because the clinically significant change score for the ASBQ has not been determined, we used the expected change in the SNOT-22 score for sample-size calculation. Hopkins et al. reported a mean (standard deviation) postoperative change score of 16.2 (20.0) in 2077 patients undergoing endoscopic sinus surgery. ${ }^{14}$ Dividing this change score by the total number of 22 questions yields a mean item score of 0.74 , which is of the same magnitude as the scores reported for the ASBQ. Therefore, given a true difference between pre- and postoperative scores of 0.74 with a standard deviation of 0.91 , a total of 15 cases would be needed to detect a treatment difference using a paired t-test at a 2-sided alpha level of 0.05 and a power of $80 \%$.

Statistical analysis was performed using SPSS version 19.0 (SPSS Inc.). Each case served as its own historical control. Mean preoperative and postoperative scores were compared using the paired t-test. An unpaired t-test was used in univariate analyses. All $\mathrm{p}$ values were 2 -sided with statistical significance evaluated at the 0.05 alpha level. Ninety-five percent confidence intervals were constructed to assess the precision of the obtained estimates.

\section{Results}

Eighty-one patients met the criteria for study enrollment. Forty-five (55.6\%) were female and $36(44.4 \%)$ were male, with a mean (SD) age of 53.1 (16.9) years. Median follow-up was 16 months with a range of 7-43 months.

Tumor characteristics are given in Table 1. Sixty-two (76.5\%) tumors were macroadenomas and 19 (23.4\%) were microadenomas. Endocrine hypersecretion was present in $57(70.4 \%)$ tumors, and visual impairment was present in $39(48.1 \%)$ patients. Cavernous sinus invasion was present in $19(23.5 \%)$ patients and suprasellar extension was present in $34(42.0 \%)$. Prior attempts at pituitary resection had been made in $10(12.3 \%)$ patients.

Surgical characteristics are given in Table 2. All cases used the endoscopic transsphenoidal corridor. Gross-total resection (GTR) was achieved in $66(81.5 \%)$ cases, including $23(95.8 \%)$ of the non-hormone-producing tumors. Lumbar drains were placed intraoperatively in 29 (35.8\%) patients. Multilayer reconstruction of the surgical defect was used in all cases, including autologous fat or fascia lata grafting in $33(40.7 \%)$ and a vascularized nasoseptal flap in 47 (58.0\%). Major postoperative complications such as CSF leakage and meningitis were not encountered in this series.

Paired analysis of overall ASBQ scores at 3 weeks postoperatively indicated decreased QOL compared with preoperative scores (Fig. 1). At 6 weeks after surgery, there was no difference between preoperative and postoperative scores. A significant improvement in overall ASBQ scores was present at 12 weeks, 6 months, and 1 year following surgery $(\mathrm{p}<0.05)$.

Independent analysis of the ASBQ components revealed significant improvements in QOL in 5 of 6 individual domains (Fig. 2). The domains of performance, physical function, and vitality showed significant improvement beginning at 12 weeks postoperatively, while pain and emotional impact showed significant improvement beginning 6 weeks after surgery. Only the specific symptoms

TABLE 1. Tumor characteristics from patients undergoing endoscopic resection of pituitary adenoma

\begin{tabular}{cc}
\hline Characteristic & No. $(\%)$ \\
\hline Anatomical site & \\
\hline Sella & $81(100)$ \\
\hline Suprasellar cistern & $34(42.0)$ \\
\hline Cavernous sinus & $19(23.5)$ \\
\hline Endocrinological & \\
\hline Hypersecreting & $57(70.4)$ \\
\hline Nonsecreting & $24(29.6)$ \\
\hline Visual deficit & $39(48.1)$ \\
\hline Prior surgery & $10(12.3)$ \\
\hline
\end{tabular}


TABLE 2. Surgical characteristics from patients undergoing endoscopic resection of pituitary adenoma

\begin{tabular}{cc}
\hline Characteristic & No. (\%) \\
\hline Corridor & $81(100)$ \\
\hline Transsphenoidal & $4(6.2)$ \\
\hline Transethmoidal & $1(1.5)$ \\
\hline Transmaxillary & \\
\hline Resection status & $66(81.5)$ \\
\hline GTR & $15(18.5)$ \\
\hline STR & $0(0)$ \\
\hline CSF leak & $51(63.0)$ \\
\hline Preop & $0(0)$ \\
\hline Intraop & \\
\hline Postop & $47(58.0)$ \\
\hline Closure & $7(8.6)$ \\
\hline Nasoseptal flap & $33(40.7)$ \\
\hline Gasket seal & \\
\hline Fat & $29(35.8)$ \\
\hline Lumbar drain & $0(0)$ \\
\hline Intraop & $33(40.7)$ \\
\hline Postop & \\
\hline Donor site & \\
\hline
\end{tabular}

domain showed transient worsening at 3 weeks postoperatively, but by 6 months after surgery these scores had improved to above preoperative values. This latter trend mirrored the effect on sinonasal-related QOL as assessed by the SNOT-22, whose scores worsened at 3 weeks after surgery with subsequent improvement to preoperative levels (Fig. 3).

Univariate analysis was performed on ASBQ score trends (Fig. 4). Improved postoperative QOL was observed beginning 12 weeks postoperatively for patients who underwent GTR compared with those who had subtotal resection (STR) $(\mathrm{p}<0.05)$. The presence of suprasellar extension or cavernous sinus invasion was not associated with worse postoperative QOL. Similarly, the occurrence of an intraoperative CSF leak or the type of reconstruction were not associated with differences in QOL at any time point.

Comparable QOL was reported by patients with and

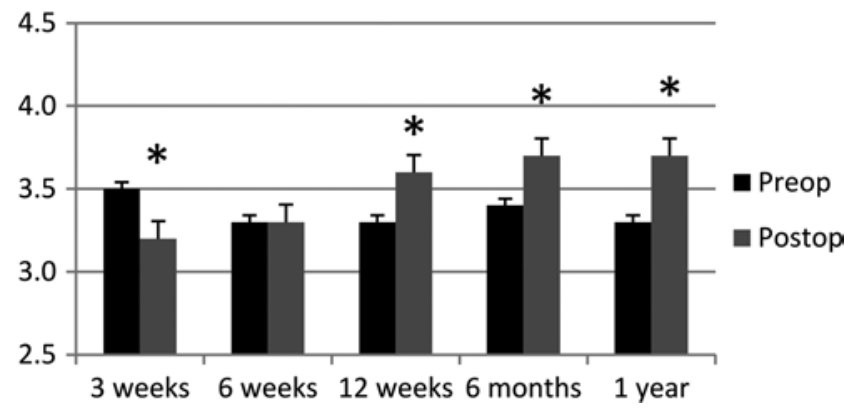

FIG. 1. Preoperative and postoperative mean overall $A S B Q$ scores for patients who underwent endoscopic resection of pituitary adenoma. The $y$-axis represents the scores. A higher score indicates a better QOL. * $p$ $<0.05$, paired 2-tailed t-test. without a preoperative visual deficit. Among patients with preoperative deficit, postoperative improvement occurred in 17 of 39 cases $(43.6 \%)$. There was no significant difference in QOL between patients with improved postoperative vision and those with stable postoperative vision. Preoperative QOL was comparable for patients with secreting and nonsecreting tumors, and postoperative QOL was not significantly different at any time point. Among nonsecreting tumors, GTR was achieved in 23 of 24 cases (95.8\%). Among secreting tumors, postoperative remission occurred in 21 of 57 cases (36.8\%). Improvement in QOL was independent of the occurrence of endocrine remission. Suppressive medical management was employed postoperatively in cases of surgical failure. While the success of such therapy might improve QOL, these cases would still be considered surgical failures and the impact of that categorization on patient psychology would remain the same. Patients with secreting tumors treated using STR had significantly worse QOL at multiple time points following surgery (Fig. 5).

\section{Discussion}

As endoscopic and other minimal-access surgery continues to gain popularity in clinical practice, surgeons are faced with the challenge of defining the benefit of their new techniques with regard to patient-related outcomes. Pituitary adenomas are one of the most common benign tumors treated by neurosurgeons, and the recent paradigm shift from microscopic to endoscopic surgery is still evolving. Meta-analyses have suggested that operative time is comparable between the 2 approaches and that endoscopic surgery may be associated with shorter hospitalizations and fewer complications. ${ }^{5,18}$

The principal finding of this current prospective study is that endoscopic surgery is associated with improved site-specific (skull base) QOL up to 1 year following surgery. This finding suggests that minimal-access surgery for this common pathology not only maintains QOL but can improve it compared with preoperative levels. This effect on QOL is consistent with findings in a previous prospective study that examined QOL after endoscopic skull base surgery for a heterogeneous group of pathologies. This previous study showed improved site-specific QOL beginning 12 weeks after surgery for a variety of skull base pathologies. ${ }^{21}$ Factors that underlie this finding may include the relief of postoperative anxiety and the benign nature of pituitary tumors, which are generally associated with minimal postoperative neurological deficits and overall improvement in preoperative symptoms.

Analysis of individual domains provides additional information about the effects of endoscopic surgery. Five of the 6 ASBQ domains demonstrated early significant improvements after surgery compared with preoperative values. The emotional impact domain showed a favorable trend as early as 6 weeks postoperatively, which may reflect the relief of preoperative anxiety. The patient's perception of pain domain also improved beginning at 6 weeks. Since pituitary adenoma is not typically associated with pain, this finding likely reflects the resolution of perioperative discomfort. The performance, physical function, 

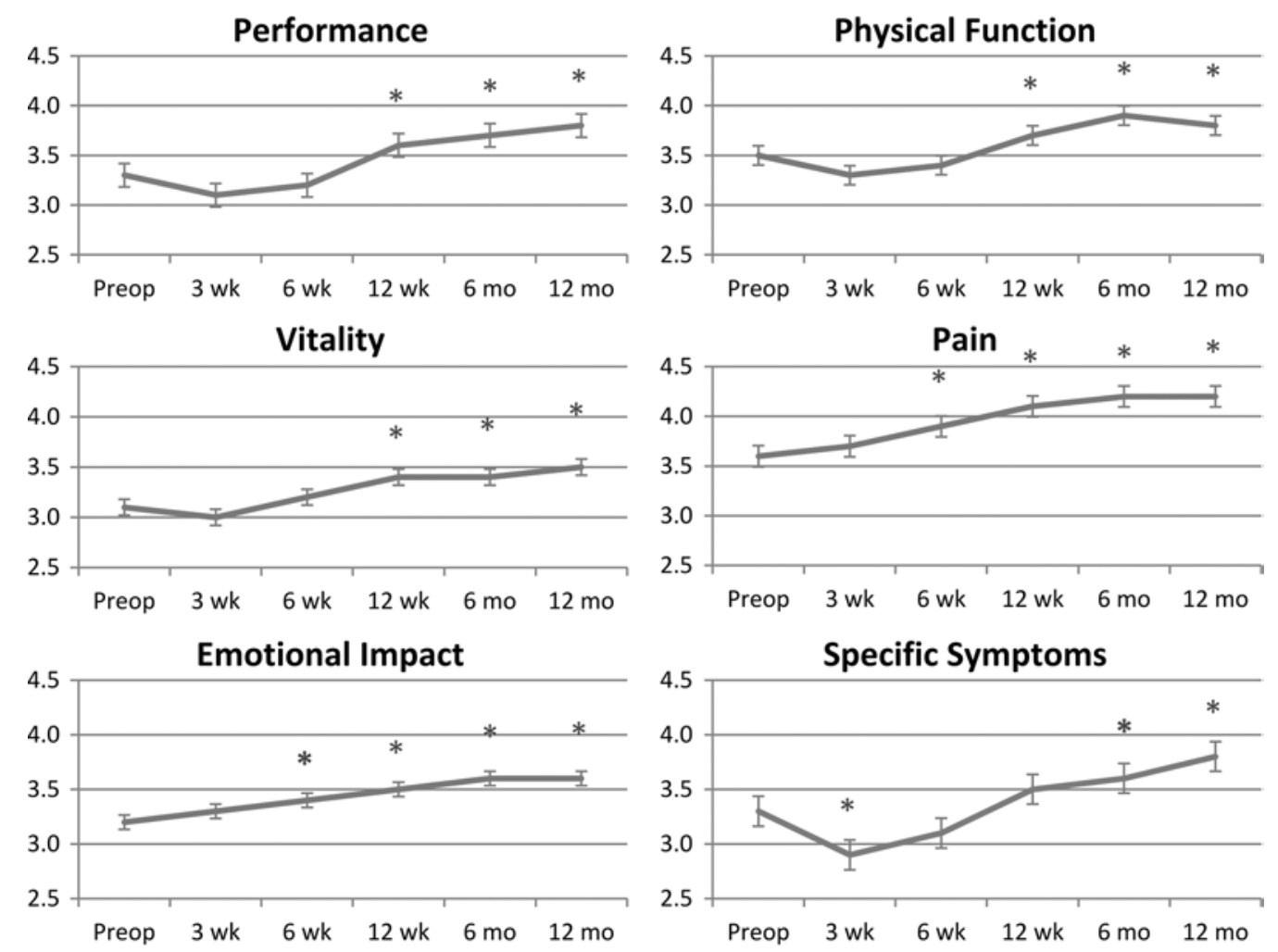

FIG. 2. Individual domains assessed by the $A S B Q$ showing preoperative and postoperative mean scores for patients who underwent endoscopic pituitary surgery. The y-axes represent the scores. A higher score indicates a better QOL. ${ }^{*} p<0.05$, paired 2-tailed t-test.

and vitality domains showed postoperative improvement at 12 weeks. Each of these domains includes aspects of well-being that reflect a favorable recovery time and low rate of complications. The last domain, which assesses the effect on specific symptoms, showed a transient worsening at 3 weeks postoperatively. This finding reflects the expected presence of intranasal edema, crusting, and hyposmia that accompanies endonasal surgery in the early postoperative period. That this domain returned to baseline at 6 weeks and ultimately showed improvement at 6 months supports the resolution of these physical effects. This latter trend is supported by the postoperative changes observed in SNOT-22 scores, which provide a detailed assessment of sinonasal QOL and indicate a transient wors-

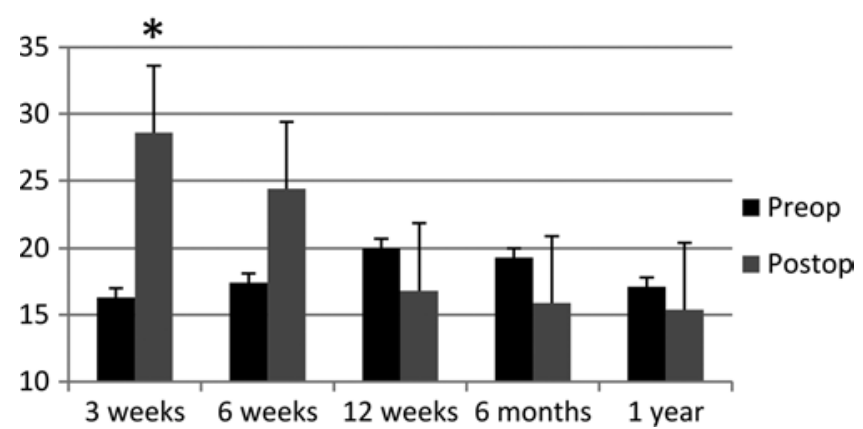

FIG. 3. Sinonasal-related QOL showing mean preoperative and postoperative SNOT-22 scores for patients who underwent endoscopic pituitary surgery. The y-axis represents the scores. A lower score indicates a better QOL. ${ }^{*} p<0.05$, paired 2-tailed t-test. ening of QOL followed by a return to baseline in the nearterm postoperative period.

GTR was achieved with endoscopic surgery in the majority of patients in this series, which was associated with a better postoperative QOL than that in patients who had STR. This may reflect a favorable sense of well-being by the patient who considers the surgery to be a success. In addition, patients with STR may be preparing to undergo radiotherapy or additional surgery, which may contribute to a worse QOL. Among hypersecreting tumors in this study, GTR was associated with improved postoperative QOL, which supports the importance of the goal of complete resection in such cases. Similar analysis of nonsecreting tumors was not possible because nearly all were gross-totally removed. These data indicate the importance of pursuing a GTR to increase a patient's postoperative QOL. Endoscopic surgery provides improved visualization, and expanded endoscopic skull base techniques improve exposure and the possibility of achieving a more complete resection, without increasing morbidity as reflected in QOL outcome. ${ }^{12}$

Tumor involvement of the suprasellar cistern and cavernous sinus were present in over $42 \%$ and $23.5 \%$ of cases, respectively, in this series. Neither factor was associated with worse preoperative QOL nor with deterioration of postoperative QOL as compared with cases where the tumor was confined to the sella. This important finding supports the feasibility and suitability of endoscopic techniques for resection of tumors with extrasellar extension. Using the endoscopic technique for removal of these tu- 
Suprasellar Extension

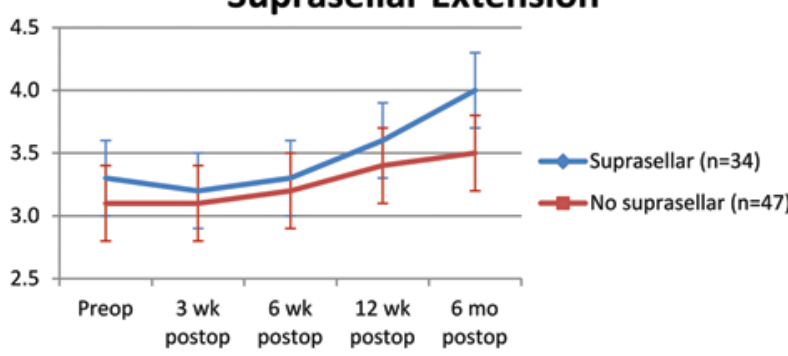

Cavernous Sinus Invasion

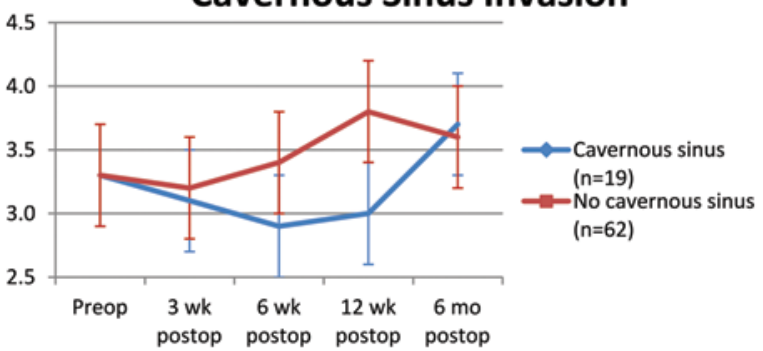

Degree of Resection

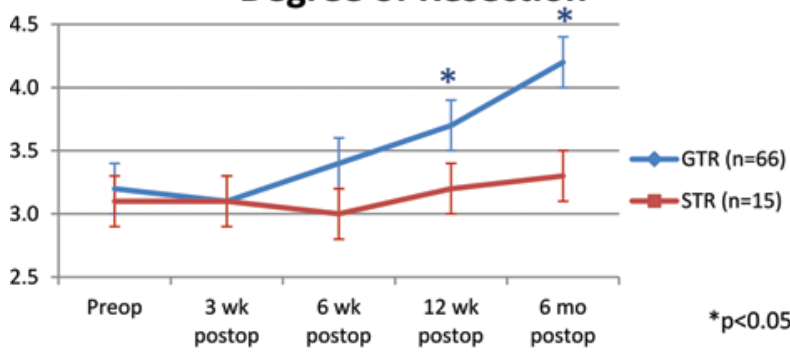

Hormone Secreting

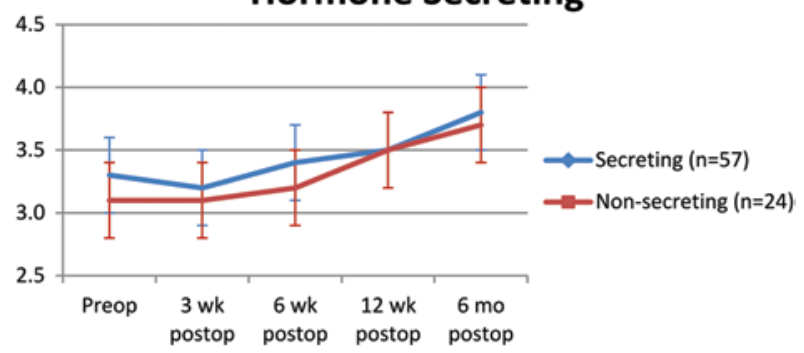

Nasoseptal Flap

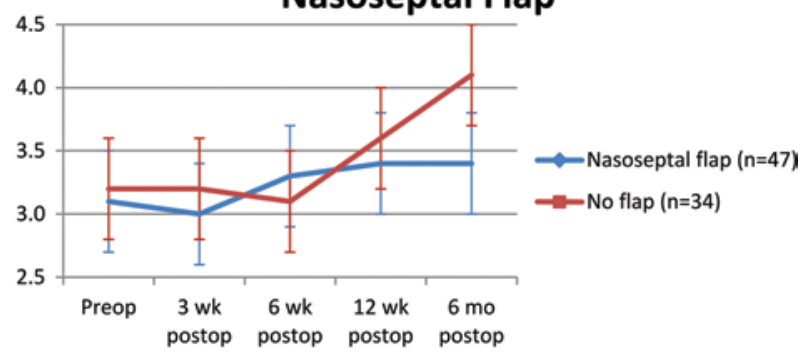

Donor Site

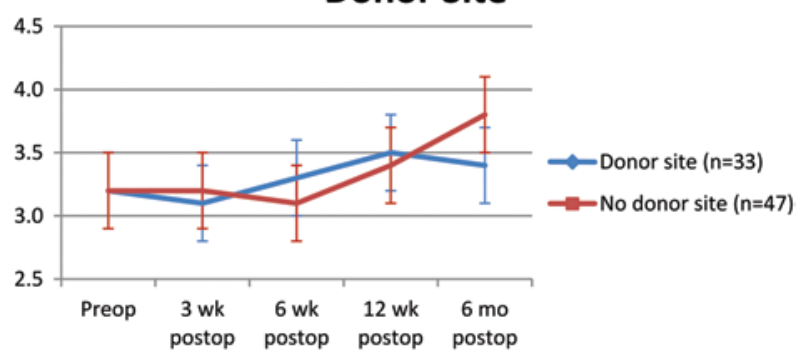

FIG. 4. Univariate analysis of $A S B Q$ scores for patients who underwent endoscopic pituitary surgery. A higher score indicates a better QOL. The y-axes represent the scores. ${ }^{*} p<0.05$, 2-tailed t-test. Figure is available in color online only.

mors may also avoid the decreased QOL that may result from traditional surgical approaches. Previous research has validated the use of the ASBQ to assess endoscopic surgical outcomes, with $75 \%$ of cases reporting improvement or no change in overall QOL. ${ }^{4}$

Reconstruction of the postoperative skull base defect is an important consideration in all cases regardless of surgical approach. Achieving low rates of postoperative CSF leak following endoscopic surgery has been well demonstrated with more recent publications in the literature. ${ }^{27}$ Results of the current study support previous findings that site-specific QOL is not impacted by the type of reconstruction, including the use of a vascularized nasoseptal flap..$^{20}$ Other studies have reported transient impairment of mucociliary clearance time and subjective olfaction after nasoseptal flap reconstruction, although the clinical significance of those findings is not clear and similar impairment has been reported after pituitary surgery without flap reconstruction. ${ }^{1,2}$ Similarly, the presence of a donor site for harvesting autologous graft material is not associated with worse postoperative QOL. Taken together, these findings illustrate that skull base reconstruction considerations should not prevent surgeons from applying the endoscopic approach for pituitary resection and that harvesting and using a nasoseptal flap in large tumors, where a significant CSF leak is expected, is a well-tolerated procedure.
Several limitations of this study bear mention. Smaller sample sizes were obtained for later time points, which primarily reflect ongoing data collection. However, the sample size at each point was adequately powered to detect a true difference between paired preoperative and postoperative scores. The absence of a control group leads to the risk of observation bias and performance bias. Recruitment of patients from a single center raises the potential for selection bias. Data on certain potential confounding factors such as socioeconomic status and educational level were not collected and therefore were not controlled for in this analysis. Sinonasal morbidity, as reflected by the SNOT-22 and the specific symptoms domains, may have been adversely affected by the inclusion of partial inferior turbinectomy in all cases. Omission of this procedure may manifest as additional gains in sinonasal and overall QOL. Most importantly, the present study represents outcomes research that can identify clinical associations but cannot exclude the possibility of confounding factors. This methodology is acceptable given the logistical and ethical limitations of experimental research on surgical interventions. Nonetheless, direct comparison of endoscopic with open approaches using validated QOL measures will be necessary to ultimately determine the superiority of one approach over the other with respect to this outcome parameter. 


\section{Endocrine Improvement}

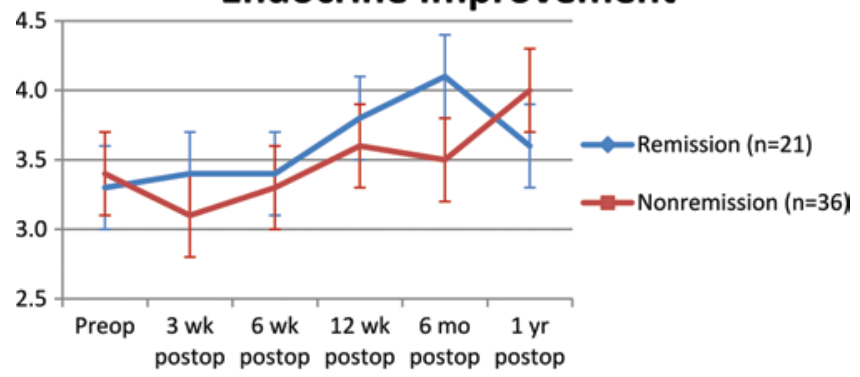

Degree of Resection

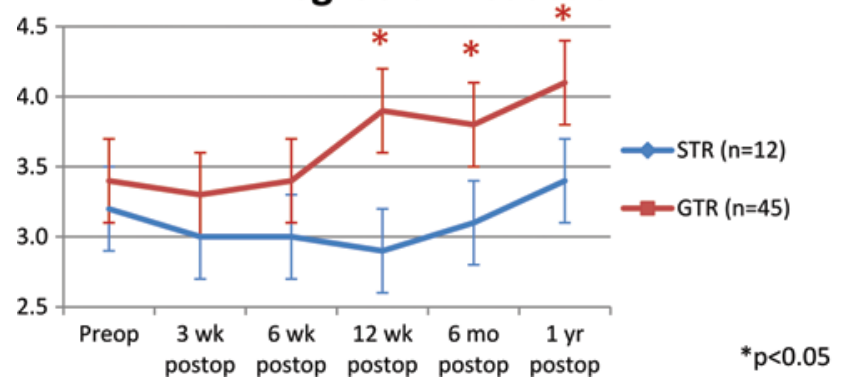

FIG. 5. Univariate analysis of ASBQ scores according to endocrine function. A higher score indicates a better QOL. Endocrine remission among hypersecreting tumors (upper). Effect of resection status among hypersecreting tumors (lower). The $y$-axes represent the scores. ${ }^{*} p<0.05$, 2-tailed t-test. Figure is available in color online only.

\section{Conclusions}

Endoscopic resection of pituitary adenoma is associated with improved site-specific QOL as the time after surgery increases. The use of validated instruments in a prospective study design with paired data provides assurance that true effects are being observed. Multiple individual QOL domains show long-term improvement, while sinonasal symptoms show transient worsening during the early postoperative period. Although STR is associated with worse postoperative QOL, extrasellar tumor extension and functional secretion do not reduce postoperative QOL. Preservation and/or improvement of patient-reported QOL are important goals when selecting the surgical approach for resection of pituitary adenoma, and comparisons with microscope-based surgeries and medical or radiation therapy are warranted.

\section{References}

1. Alobid I, Enseñat J, Mariño-Sánchez F, de Notaris M, Centellas S, Mullol J, et al: Impairment of olfaction and mucociliary clearance after expanded endonasal approach using vascularized septal flap reconstruction for skull base tumors. Neurosurgery 72:540-546, 2013

2. Alobid I, Enseñat J, Mariño-Sánchez F, Rioja E, de Notaris M, Mullol J, et al: Expanded endonasal approach using vascularized septal flap reconstruction for skull base tumors has a negative impact on sinonasal symptoms and quality of life. Am J Rhinol Allergy 27:426-431, 2013

3. Brown SM, Anand VK, Tabaee A, Schwartz TH: Role of perioperative antibiotics in endoscopic skull base surgery. Laryngoscope 117:1528-1532, 2007

4. Cavel O, Abergel A, Margalit N, Fliss DM, Gil Z: Quality of life following endoscopic resection of skull base tumors. J Neurol Surg B Skull Base 73:112-116, 2012
5. DeKlotz TR, Chia SH, Lu W, Makambi KH, Aulisi E, Deeb Z: Meta-analysis of endoscopic versus sublabial pituitary surgery. Laryngoscope 122:511-518, 2012

6. Georgalas C, Badloe R, van Furth W, Reinartz S, Fokkens WJ: Quality of life in extended endonasal approaches for skull base tumours. Rhinology 50:255-261, 2012

7. Gil Z, Abergel A, Spektor S, Cohen JT, Khafif A, Shabtai E, et al: Quality of life following surgery for anterior skull base tumors. Arch Otolaryngol Head Neck Surg 129:13031309,2003

8. Gil Z, Abergel A, Spektor S, Khafif A, Fliss DM: Patient, caregiver, and surgeon perceptions of quality of life following anterior skull base surgery. Arch Otolaryngol Head Neck Surg 130:1276-1281, 2004

9. Gil Z, Abergel A, Spektor S, Shabtai E, Khafif A, Fliss DM: Development of a cancer-specific anterior skull base qualityof-life questionnaire. J Neurosurg 100:813-819, 2004

10. Graham SM, Iseli TA, Karnell LH, Clinger JD, Hitchon PW, Greenlee JDW: Endoscopic approach for pituitary surgery improves rhinologic outcomes. Ann Otol Rhinol Laryngol 118:630-635, 2009

11. Hofstetter CP, Mannaa RH, Mubita L, Anand VK, Kennedy JW, Dehdashti AR, et al: Endoscopic endonasal transsphenoidal surgery for growth hormone-secreting pituitary adenomas. Neurosurg Focus 29(4):E6, 2010

12. Hofstetter CP, Nanaszko MJ, Mubita LL, Tsiouris J, Anand VK, Schwartz TH: Volumetric classification of pituitary macroadenomas predicts outcome and morbidity following endoscopic endonasal transsphenoidal surgery. Pituitary 15:450-463, 2012

13. Hofstetter CP, Shin BJ, Mubita L, Huang C, Anand VK, Boockvar JA, et al: Endoscopic endonasal transsphenoidal surgery for functional pituitary adenomas. Neurosurg Focus 30(4):E10, 2011

14. Hopkins C, Gillett S, Slack R, Lund VJ, Browne JP: Psychometric validity of the 22 -item Sinonasal Outcome Test. Clin Otolaryngol 34:447-454, 2009

15. Jakimovski D, Bonci G, Attia M, Shao H, Hofstetter C, Tsiouris AJ, et al: Incidence and significance of intraoperative cerebrospinal fluid leak in endoscopic pituitary surgery using intrathecal fluorescein. World Neurosurg 82: e513e523, 2014

16. Johnson MD, Woodburn CJ, Vance ML: Quality of life in patients with a pituitary adenoma. Pituitary 6:81-87, 2003

17. Kirkman MA, Borg A, Al-Mousa A, Haliasos N, Choi D: Quality-of-life after anterior skull base surgery: a systematic review. J Neurol Surg B Skull Base 75:73-89, 2014

18. Komotar RJ, Starke RM, Raper DM, Anand VK, Schwartz TH: Endoscopic endonasal compared with microscopic transsphenoidal and open transcranial resection of giant pituitary adenomas. Pituitary 15:150-159, 2012

19. Lwu S, Edem I, Banton B, Bernstein M, Vescan A, Gentili F, et al: Quality of life after transsphenoidal pituitary surgery: a qualitative study. Acta Neurochir (Wien) 154:1917-1922, 2012

20. McCoul ED, Anand VK, Bedrosian JC, Schwartz TH: Endoscopic skull base surgery and its impact on sinonasal-related quality of life. Int Forum Allergy Rhinol 2:174-181, 2012

21. McCoul ED, Anand VK, Schwartz TH: Improvements in site-specific quality of life 6 months after endoscopic anterior skull base surgery: a prospective study. J Neurosurg 117:498-506, 2012

22. McCoul ED, Anand VK, Singh A, Nyquist GG, Schaberg MR, Schwartz TH: Long-term effectiveness of a reconstructive protocol using the nasoseptal flap after endoscopic skull base surgery. World Neurosurg 81:136-143, 2014

23. Morley AD, Sharp HR: A review of sinonasal outcome scoring systems - which is best? Clin Otolaryngol 31:103-109, 2006 
24. Piccirillo JF, Merritt MG Jr, Richards ML: Psychometric and clinimetric validity of the 20-Item Sino-Nasal Outcome Test (SNOT-20). Otolaryngol Head Neck Surg 126:41-47, 2002

25. Schwartz TH, Fraser JF, Brown S, Tabaee A, Kacker A, Anand VK: Endoscopic cranial base surgery: classification of operative approaches. Neurosurgery 62:991-1005, 2008

26. Suberman TA, Zanation AM, Ewend MG, Senior BA, Ebert CS Jr: Sinonasal quality-of-life before and after endoscopic, endonasal, minimally invasive pituitary surgery. Int Forum Allergy Rhinol 1:161-166, 2011

27. Tabaee A, Anand VK, Barrón Y, Hiltzik DH, Brown SM, Kacker A, et al: Endoscopic pituitary surgery: a systematic review and meta-analysis. J Neurosurg 111:545-554, 2009

28. Tabaee A, Placantonakis DG, Schwartz TH, Anand VK: Intrathecal fluorescein in endoscopic skull base surgery. Otolaryngol Head Neck Surg 137:316-320, 2007

29. Tanemura E, Nagatani T, Aimi Y, Kishida Y, Takeuchi K, Wakabayashi T: Quality of life in nonfunctioning pituitary macroadenoma patients before and after surgical treatment. Acta Neurochir (Wien) 154:1895-1902, 2012

30. Wang YY, Srirathan V, Tirr E, Kearney T, Gnanalingham KK: Nasal symptoms following endoscopic transsphenoidal pituitary surgery: assessment using the General Nasal Patient Inventory. Neurosurg Focus 30(4):E12, 2011

\section{Author Contributions}

Conception and design: Schwartz, McCoul, Anand. Acquisition of data: all authors. Analysis and interpretation of data: Schwartz, McCoul, Bedrosian, Anand. Drafting the article: McCoul. Critically revising the article: all authors. Reviewed submitted version of manuscript: all authors. Statistical analysis: McCoul. Study supervision: Schwartz, Anand.

\section{Supplemental Information}

Previous Presentation

Portions of this work were presented at the North American Skull Base Society Annual Meeting held in Las Vegas, Nevada, on February 17-19, 2012.

\section{Correspondence}

Theodore H. Schwartz, Department of Neurological Surgery, Weill Cornell Medical College, NewYork-Presbyterian Hospital, 525 E. 68th St., New York, NY 10065. email: schwarh@med. cornell.edu. 